\title{
Discrimination of tropical forest types, dominant species, and mapping of functional guilds by hyperspectral and simulated multispectral Sentinel-2 data
}

\author{
Gaia Vaglio Laurin ${ }^{\mathrm{a}, \mathrm{b}, *}$, Nicola Puletti ${ }^{\mathrm{c}}$, William Hawthorne ${ }^{\mathrm{d}}$, Veraldo Liesenberg ${ }^{\mathrm{e}}$, Piermaria Corona ${ }^{\mathrm{c}}$, \\ Dario Papale ${ }^{\mathrm{b}}$, Qi Chen ${ }^{\mathrm{f}}$, Riccardo Valentini ${ }^{\mathrm{a}, \mathrm{b}}$ \\ a Impacts of Agriculture, Forests and Ecosystem Services Division, Euro-Mediterranean Center on Climate Change (IAFES-CMCC), via Pacinotti 5, Viterbo 01100, Italy \\ b Department for Innovation in Biological, Agro-Food and Forest Systems (DIBAF), University of Tuscia, Viterbo 01100, Italy \\ c Consiglio per la ricerca in agricoltura e l'analisi dell'economia agraria, Forestry Research Centre (CRA-SEL), Via Santa Margherita 80, I-52100 Arezzo, Italy \\ d Department of Plant Sciences, University of Oxford, South Parks Road, Oxford OX1 3RB, UK \\ e Santa Catarina State University (UDESC), Av. Luiz de Camoes, 2090, Lages, Santa Catarina 88520-000, Brazil \\ f Department of Geography, University of Hawai'i at Mānoa, 422 Saunders Hall, 2424 Maile Way, Honolulu, HI 96822, USA
}

\section{A R T I C L E I N F O}

\section{Article history:}

Received 13 June 2015

Received in revised form 13 January 2016

Accepted 21 January 2016

Available online $\mathrm{xxxx}$

\section{Keywords:}

Dominant species

Forest

Forest type

Functional composition

Guilds

Hyperspectral

Tropical

Sentinel-2

\begin{abstract}
A B S T R A C T
To answer new scientific and ecological questions and monitor multiple forest changes, a fine scale characterization of these ecosystems is needed, and could imply the mapping of specific species, of detailed forest types, and of functional composition. This characterization can be now provided by the novel Earth Observation tools. This study aims to contribute to understanding the innovation in forest and ecological research that can be brought in by advanced remote sensing instruments, and proposes the guild mapping approach as a tool to efficiently monitor the varied tropical forest resources. We evaluated, in tropical Ghanaian forests, the ability of airborne hyperspectral and simulated multispectral Sentinel-2 data, and derived vegetation indices and textures, to: distinguish between two different forest types; to discriminate among selected dominant species; and to separate trees species grouped according to their functional guilds: Pioneer, Non Pioneer Light Demanding, and Shade Bearer. We then produced guild classification maps for each area using hyperspectral data. Our results showed that with both hyperspectral and simulated Sentinel-2 data these discrimination tasks can be successfully accomplished. Results also stressed the importance of texture features, especially if using the lower spectral and spatial Sentinel-2 resolution data, and highlighted the important role of the new Sentinel-2 data for ecological monitoring. Classification results showed a statistically significant improvement in overall accuracy using Support Vector Machine, over Maximum Likelihood approach. We proposed the functional guilds mapping as an innovative approach to: (i) monitor compositional changes, especially with respect to the effects of global climate change on forests, and particularly in the tropical biome where the occurrence of hundreds of species prevents mapping activities at species level; (ii) support large-scale forest inventories. The imminent Sentinel-2 data could serve to open the road for the development of new concepts and methods in forestry and ecological research.
\end{abstract}

(c) 2016 Elsevier Inc. All rights reserved.

\section{Introduction}

Tropical forests host the largest biodiversity of terrestrial ecosystems and have a fundamental role in the carbon cycle. Improving the monitoring of tropical forests is an important research issue, relevant to the implementation of climate change related agreements and reporting duties, to biodiversity conservation, and to the definition of sustainable schemes for timber extraction. The understanding of ecological mechanisms can also benefit from improved forest monitoring, as in the case

\footnotetext{
* Corresponding author at: Impacts of Agriculture, Forests and Ecosystem Services Division, Euro-Mediterranean Center on Climate Change (IAFES-CMCC), via Pacinotti 5 , Viterbo 01100, Italy.

E-mail address: gaia.vagliolaurin@cmcc.it (G. Vaglio Laurin).
}

of the dynamics of tree species distribution in different ecosystems, community structures, and spatial distributions of functional traits. For both applied and scientific purposes, the use of Earth Observation (EO) is fundamental, allowing extrapolation of local field information, difficult to collect, to the large extents typical of tropical forests. EO systems that allow for credible measurement, reporting and verification are particularly critical for the successful implementation of REDD + (Reducing Emissions from Deforestation and Forest Degradation) efforts by the United Nations (UN-REDD, 2013).

The initial remote sensing focus in forest research, a few decades ago, pointed toward the detection of deforestation and forest land conversion, and the coarse characterization of different forest types. These tasks have been successfully accomplished using optical data, which are now a consolidated tool to monitor, at large and medium scales, 